\title{
Heart transplant graft survival is improved after a reduction in panel reactive antibody activity
}

\author{
Justin M. Schaffer, MD, ${ }^{\mathrm{a}}$ Steve K. Singh, MD, ${ }^{\mathrm{b}}$ Bruce A. Reitz, MD, ${ }^{\mathrm{a}}$ Philip E. Oyer, MD, ${ }^{\mathrm{a}}$ \\ Robert C. Robbins, MD, ${ }^{a}$ and Hari R. Mallidi, MD $^{\mathrm{c}}$
}

\begin{abstract}
Background: Allosensitization in potential orthotopic heart transplant recipients is evaluated with the panel reactive antibody assay. Sensitized patients have prolonged wait times and increased waitlist and posttransplant mortality. Although low panel reactive antibody activity at the time of orthotopic heart transplantation is associated with improved outcomes, literature regarding the survival benefit of a panel reactive antibody reduction in the sensitized orthotopic heart transplant recipient remains limited.
\end{abstract}

Methods: Adult orthotopic heart transplant recipients listed in the United Network for Organ Sharing database (October 1, 1987, to June 29, 2004) were stratified by peak panel reactive antibody activity and whether a substantial decline from peak to most recent panel reactive antibody activity occurred before transplant. Propensity matching adjusted for differences in recipient and donor characteristics. Graft survival was assessed with Kaplan-Meier analysis. Cox proportional hazards regression determined predictors of graft survival.

Results: Pretransplant characteristics differed between sensitized patients who had a substantial decline in panel reactive antibody activity and those who did not. Propensity matching compensated for these differences. Kaplan-Meier survival analysis of matched groups showed that the median graft survival was 120 months in patients with a significant panel reactive antibody reduction and 103 months in patients with a trivial reduction $(P=.007, \log$-rank). In Cox proportional hazards modeling, a significant reduction in panel reactive antibody activity had an independent protective effect on graft survival (hazard ratio, 0.88 ; confidence interval, 0.80-0.96; $P=.006)$.

Conclusions: Sensitized patients who had a substantial reduction in panel reactive antibody activity had an associated decline in the incidence of graft failure compared with those without a panel reactive antibody activity reduction. These results support efforts to reduce panel reactive antibody activity before orthotopic heart transplantation in patients with high panel reactive antibody activity. (J Thorac Cardiovasc Surg 2013;145:555-65)

Allosensitization is a condition in which recipient serum antibodies react against donor antigens. ${ }^{1}$ It can be directly assessed by the donor-specific crossmatch, wherein recipient serum is mixed with antigen derived from donor leukocytes to determine cross-reactivity, a so-called "cell-based" approach. $^{2}$ As initially shown in kidney transplantation, a positive donor-specific crossmatch is associated with decreased graft survival after orthotopic heart transplantation $(\mathrm{OHT}){ }^{3,4}$ Although retrospective crossmatches are performed for OHT recipients in the majority of centers,

\footnotetext{
From the Department of Cardiothoracic Surgery, ${ }^{\text {a }}$ Stanford Hospital and Clinics, Stanford, Calif; Division of Cardiothoracic Surgery, ${ }^{\mathrm{b}}$ Department of Surgery, Hamilton General Hospital, Hamilton, Ontario, Canada; and Division of Cardiothoracic Surgery, ${ }^{\mathrm{c}}$ Department of Surgery, Baylor College of Medicine, and the Center for Cardiac Support, Texas Heart Institute, Houston, Tex.

Disclosures: Authors have nothing to disclose with regard to commercial support.

Read at the 38th Annual Meeting of The Western Thoracic Surgical Association, Maui, Hawaii, June 27-30, 2012.

Received for publication June 28, 2012; revisions received Sept 21, 2012; accepted for publication Oct 15, 2012; available ahead of print Dec 13, 2012.

Address for reprints: Hari R. Mallidi, MD, Baylor College of Medicine, Texas Heart Institute at St Luke's Episcopal Hospital, 6770 Bertner Ave, MC2-114A, Houston, TX 77030 (E-mail: mallidi@bcm.edu).

0022-5223/\$36.00

Copyright (C) 2013 Published by Elsevier Inc. on behalf of The American Association for Thoracic Surgery

http://dx.doi.org/10.1016/j.jtcvs.2012.10.025
}

the need for a source of both donor leukocytes and recipient serum to perform the assay, the cold ischemic time constraint associated with OHT, and the low sensitivity and specificity of the assay make prospectively crossmatching all OHT recipients a near impossibility. ${ }^{5,6}$ Instead, allosensitization is prospectively assessed by the panel reactive antibody (PRA) test, wherein an intended recipient's serum is exposed to several different human leukocyte antigen (HLA)-typed lymphocytes representing theoretic donors (again, a cell-based approach) to assess HLA cross-reactivity; the percentage of theoretic donor cells that react is used as a surrogate for sensitization. ${ }^{1}$ In OHT recipients, as was first shown in kidney recipients, elevated PRA activity is associated with decreased graft survival; this association is independent of the crossmatch result. ${ }^{4,7-9}$

Despite numerous modifications-including wash steps to remove anti-complimentary factors, increased incubation times, and the addition of anti-human immunoglobulin to facilitate binding of low-avidity, low-titer, and noncomplement fixing antibodies - cell-based approaches remain limited by their relatively poor sensitivity and specificity, the arbitrary composition of target cell panels, issues related to cell viability, and the restriction of cell-based tests to detecting class I antibodies only. ${ }^{10-12}$ The recent introduction 


$$
\begin{aligned}
& \text { Abbreviations and Acronyms } \\
& \text { BMI = body mass index } \\
& \text { CI }=\text { confidence interval } \\
& \text { ECMO }=\text { extracorporeal membrane oxygenation } \\
& \text { EPY }=\text { events per patient year } \\
& \text { HLA }=\text { human leukocyte antigen } \\
& \text { HR }=\text { hazard ratio } \\
& \text { MHC }=\text { major histocompatibility complex } \\
& \text { OHT }=\text { orthotopic heart transplantation } \\
& \text { PRA }=\text { panel reactive antibody } \\
& \text { UNOS }=\text { United Network for Organ Sharing } \\
& \text { VAD }=\text { ventricular assist device }
\end{aligned}
$$

of solid-phase assays, in particular single-antigen HLA beads (HLA class I or II antigens produced by recombinant DNA technologies and attached to inert microparticles), has addressed many of the limitations of cell-based approaches. ${ }^{6,12}$ These highly sensitive and specific assays have fostered the development of the virtual crossmatch and calculated PRA tests, in which recipient serum antiHLA specificities derived from solid-phase assays are compared with the HLA antigen profile of a specific donor (virtual crossmatch) or HLA antigen frequencies in a sample donor pool (calculated PRA). ${ }^{13,14}$

Regardless of the assay used, elevated PRA activity had dire implications for potential recipients because it limits the acceptable donor pool and prolongs waitlist times, thereby increasing the incidence of waitlist mortality in these patients. ${ }^{15}$ Efforts to achieve a donor-recipient match in allosensitized patients involve closely monitoring PRA activity, strictly avoiding further exposure to foreign human antigens, and adopting an assortment of therapies aimed at reducing allosensitization, including plasmapheresis, intravenous immunoglobulin, rituximab, and bortezomib. ${ }^{1} \mathrm{Sev}$ eral institutional studies have shown that implementing these allosensitization-reduction strategies reduces PRA activity and waitlist durations and produces graft survivals similar to those of nonsensitized patients. ${ }^{1,16-19}$ However, there remains a dearth of literature regarding long-term graft survival in previously allosensitized patients whose level of allosensitization is significantly improved before transplant. To address this question, we reviewed the United Network for Organ Sharing (UNOS) database to assess graft survival in patients with significantly reduced allosensitization as indicated by pretransplant PRA activity.

\section{MATERIALS AND METHODS \\ Data Collection and Study Cohort}

We performed a retrospective review of deidentified data from the UNOS thoracic registry. Our institutional research ethics board waived the requirement for informed consent because no patient or center identifiers were included in the analysis. A total of 52,204 usable records of patients who underwent OHT between October 1, 1987, and December
31, 2011, were identified. Before June 30, 2004, the UNOS registry did not record PRA activity for HLA class II antigens because it was not assessed by most assays in clinical use at that time. The wide adoption of solid-phase assays by transplant centers occurred after June 30, 2004, making this early population more homogenous with respect to PRA assay. ${ }^{1}$ Moreover, as the number of patients bridged to transplant with a ventricular assist device (VAD) has increased, the effect of mechanical circulatory support on PRA activity has been well documented. ${ }^{20,21}$ Given our aim to address long-term graft survival and to avoid confounding by the various PRA assays, separate HLA class I/II PRA activity measurements, and the effect of VADs on PRA activity, we limited our study to the 27,102 adult patients who underwent transplantation before June 30, 2004 (before the registry began to distinguish between PRA HLA classes), whose peak and most recent PRA activity were documented (class I major histocompatibility complex $[\mathrm{MHC}]$ only). Specific focus was devoted to patients with a peak PRA of $0 \%(n=18,163)$ representing a nonsensitized control, patients with a peak PRA of $20 \%$ or greater and a trivial $(<5 \%)$ absolute reduction in PRA activity $(n=1016)$, and patients with a peak PRA of $20 \%$ or greater and a substantial $(\geq 20 \%)$ absolute reduction in PRA activity $(\mathrm{n}=1244)$. Of note, many patients $(\mathrm{n}=6679)$ had a modest PRA activity $(<20 \%)$ or a moderate reduction in PRA activity $(\geq 5 \%$ and $<20 \%)$; these patients were included in our Cox proportional hazards analysis but were omitted from our unmatched and matched Kaplan-Meier survival analyses to allow for a direct comparison of PRA-reduction nonresponders and responders. Patient follow-up data were available to March 31, 2012.

\section{Statistical Analysis}

Demographics and clinical status were obtained for all patients at the time of transplantation. Variables with missing values were imputed to avoid listwise deletion in our analysis. Multiple imputation was performed $(n=20)$ with a Markov chain Monte Carlo method that assumed a multivariate normal distribution for our data; the complete sets of observed values were used as covariates for prediction purposes. Analysis of variance was used to compare demographics and clinical status at the time of transplant among patients with a peak PRA activity of $0 \%$ (nonsensitized control), patients with a peak PRA activity of $20 \%$ or greater who had a trivial $(<5 \%)$ absolute reduction in PRA activity, and patients with a peak PRA activity of $20 \%$ or greater who had a significant $(\geq 20 \%)$ absolute reduction in PRA activity.

The primary outcome measure was graft survival (a composite end point of death and graft failure). The Kaplan-Meier method was used to examine graft survival over time, and the log-rank test was used to examine differences in graft survival curves. Censoring occurred for patients who were alive and had not undergone a repeat OHT at the last time point examined (administratively censored). Unadjusted survival analysis was assessed for each of the 3 groups described previously (peak PRA 0\%, trivial PRA reduction, substantial PRA reduction).

To better control for selection bias, we subsequently carried out propensity score matching. A multivariable logistic regression model was used to compute propensity scores for patients with a peak PRA of $20 \%$ or greater who had a trivial reduction $(<5 \%)$ or a significant reduction $(\geq 20 \%)$ in PRA activity in each of our 20 imputed datasets. These 20 propensity scores were then averaged and applied to our original data set. We next carried out a 1:1 propensity score matching analysis with a greedy matching algorithm (nearest neighbor matching within a caliper width of 1/4th of the standard deviation of the logit of the propensity score); patients without a match were excluded from the matched sample. After propensity matching, we compared matched patients with a trivial PRA reduction with those who had a significant reduction by using 2-sample $t$ tests for continuous variables and the chi-square or Fisher exact test for categoric variables. Kaplan-Meier survival analysis assessed graft survival for the propensity-matched cohort.

Univariate and multivariable Cox proportional hazards regression analyses were used to assess the effect of listing characteristics on graft survival. A multivariable model was constructed from variables that were both plausible 
for and predictive of mortality in our exploratory univariate analysis $(P \leq$ .20). Forward and backward stepwise selection were used to help ensure that the final model would be optimal. The final model included the following variables: age more than 60 years, African-American race, college education, private insurance, diagnosis of idiopathic dilated cardiomyopathy, diagnosis of congenital heart disease, undergoing redo heart transplantation, year of transplant, body mass index (BMI) $35 \mathrm{~kg} / \mathrm{m}^{2}$ or greater, ventilator dependence, extracorporeal membrane oxygenation (ECMO) support, creatinine clearance less than $50 \mathrm{~mL} / \mathrm{min}$, dialysis requirement, diabetes, serum bilirubin level $2 \mathrm{mg} / \mathrm{dL}$ or greater, organ ischemic time (hours), donor age, gender match between organ and recipient, peak PRA activity, and whether a substantial reduction in PRA activity was documented.

Means are presented with standard deviations, and hazard ratios (HRs) are presented with $95 \%$ confidence intervals (CIs). Analyses were conducted with STATA software (v 11SE, StataCorp LP, College Station, Tex).

\section{RESULTS}

We identified 52,204 usable patient records in the UNOS thoracic registry of patients who underwent OHT between October 1, 1987, and December 31, 2011. Of these patients, 45,592 were adults (aged $\geq 18$ years) at the time of transplant. Our analysis was limited to the 31,551 adult patients who underwent OHT before June 30, 2004 (when the UNOS thoracic registry began to distinguish between PRA major histocompatibility classes); of these, 4449 patients were excluded because their peak or most recent PRA values were not documented. Thus, a total of 27,102 patients were included in this study. The trends in peak and most recent PRA activity over time (Figure 1) are notable for an increase in PRA values over the duration of the study period.

\section{Baseline Characteristics at the Time of Transplant}

Forty-five variables detailing patient characteristics and operation-specific data are described in Table 1, stratified by whether the peak PRA activity was $0 \%(n=18,163)$, the peak PRA activity was $20 \%$ or higher but only a trivial $(<5 \%)$ absolute reduction in PRA activity was achieved

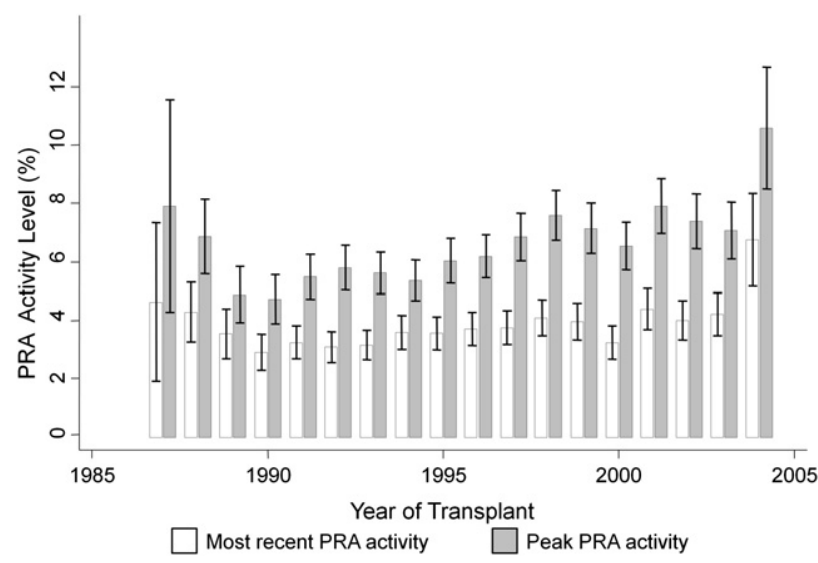

FIGURE 1. Bar graph of trends in PRA activity (both peak and most recent) at the time of transplant, stratified by year of transplant. Error bars represent $95 \%$ CIs. PRA, Panel reactive antibody. $(n=1016)$, or the peak PRA activity was $20 \%$ or higher and a substantial absolute reduction in PRA activity $(\geq 20 \%)$ was observed $(n=1244)$. The amount of missing data was significant $(<90 \%$ complete) for several variables, including level of education, insurance status, New York Heart Association functional status, creatinine clearance, dialysis requirement, diabetes, serum bilirubin, and hemodynamic parameters (ie, cardiac index, pulmonary artery pressures, and pulmonary capillary wedge pressures). As described in the "Materials and Methods," multiple imputation was used to account for missing data. Small but statistically significant differences were noted in several pretransplant and operative variables, including age at transplant, gender, diagnosis, redo OHT, BMI, listing status, New York Heart Association functional status, inotrope requirement, intra-aortic balloon pump requirement, VAD use, creatinine clearance, bicaval technique, organ ischemic time, and distance of organ transport (Table 1).

\section{Cox Proportional Hazards Analysis of Graft Survival}

Univariate and multivariable Cox proportional hazards analyses were used to identify predictors of graft survival (Table 2). A substantial reduction in PRA activity was not a predictor of graft survival on univariate analysis (HR, $1.05 ; 95 \%$ CI, $0.97-1.13 ; P=.22$ ). We suspected that having a PRA reduction was necessarily confounded by having a high peak PRA activity, and we thus included peak PRA activity in our multivariable analysis. Once multivariable analysis accounted for a patient's peak PRA activity, achieving a substantial reduction in PRA activity significantly protected against graft dysfunction (HR, 0.88; CI, $0.80-0.96 ; P=.006$ ), whereas the peak PRA activity remained an independent predictor of graft dysfunction or death (HR, 1.003; CI, 1.002-1.004; $P<.001$ ). Of note, when all 3 measures of PRA activity (peak PRA activity, most recent PRA activity, and substantial PRA activity reduction) were included in our multivariable analysis, most recent PRA activity lost statistical significance, whereas peak PRA activity and substantial PRA activity reduction remained statistically significant (Table 2).

\section{Graft Survival Analysis: Unadjusted}

The mean follow-up time from the time of transplant was $3060 \pm 2026$ days in patients who had a trivial reduction in PRA activity and $2987 \pm 1973$ days in patients who had a substantial PRA reduction. Figure 2 shows an unadjusted Kaplan-Meier estimate of graft survival from time of transplant, stratified by whether the patient had an elevated peak PRA activity and whether a trivial or significant PRA reduction was achieved; median graft survival was 131 months in patients with a peak PRA activity of $0 \%, 102$ months in patients with a peak PRA activity of $20 \%$ or greater who only had a trivial PRA reduction, and 121 months in patients with 
TABLE 1. Baseline characteristics at time of heart transplant, shown for the entire cohort, stratified by peak panel reactive antibody and panel reactive antibody reduction, and for the propensity-matched patients

\begin{tabular}{|c|c|c|c|c|}
\hline & \multicolumn{2}{|c|}{ Overall cohort } & \multicolumn{2}{|c|}{$\begin{array}{c}\text { Unadjusted analysis, stratified by peak and } \\
\text { most recent PRA activity }\end{array}$} \\
\hline & $\begin{array}{c}\text { Nonmissing data } \\
(\mathrm{n}=\mathbf{2 7 , 1 0 2})\end{array}$ & $\begin{array}{c}\text { Overall cohort } \\
(\mathrm{n}=\mathbf{2 7 , 1 0 2}) \\
\end{array}$ & $\begin{array}{c}\text { Peak PRA }=0 \%, \text { no } \\
\text { reduction in PRA } \\
(n=18,163)\end{array}$ & $\begin{array}{c}\text { Peak PRA } \geq \mathbf{2 0} \%, \\
\text { trivial reduction in } \\
\text { PRA }(<\mathbf{5} \%)(\mathrm{n}=\mathbf{1 0 1 6})\end{array}$ \\
\hline \multicolumn{5}{|l|}{ Baseline characteristics } \\
\hline Age at transplant & $27,102(100 \%)$ & $51.5 \pm 11.2$ & $51.8 \pm 11.1$ & $50.3 \pm 11.9$ \\
\hline Age $>60$ y at transplant & $27,102(100 \%)$ & $5765(21.3 \%)$ & $4000(22.0 \%)$ & $204(20.1 \%)$ \\
\hline Gender (male) & $27,102(100 \%)$ & $21,103(77.9 \%)$ & $14,414(79.4 \%)$ & $622(61.2 \%)$ \\
\hline Race (Caucasian) & $27,102(100 \%)$ & $22,134(81.7 \%)$ & $14,889(82.0 \%)$ & $796(78.4 \%)$ \\
\hline Race (African-American) & $27,102(100 \%)$ & $3106(11.5 \%)$ & $2013(11.1 \%)$ & $147(14.5 \%)$ \\
\hline Race (Hispanic) & $27,102(100 \%)$ & $1357(5.0 \%)$ & $910(5.0 \%)$ & $54(5.3 \%)$ \\
\hline Education (college) & $12,855(47.4 \%)$ & $6065(47.2 \%)$ & $4259(47.5 \%)$ & $209(43.5 \%)$ \\
\hline Insurance (private) & $18,135(66.9 \%)$ & $11,223(61.9 \%)$ & $7643(62.3 \%)$ & $425(61.2 \%)$ \\
\hline Ischemic dilated cardiomyopathy & $27,013(99.7 \%)$ & $13,425(49.7 \%)$ & $9118(50.3 \%)$ & $460(45.5 \%)$ \\
\hline Idiopathic dilated cardiomyopathy & $27,013(99.7 \%)$ & $9408(34.8 \%)$ & $6322(34.9 \%)$ & $350(34.6 \%)$ \\
\hline Valvular heart disease & $27,013(99.7 \%)$ & $822(3.0 \%)$ & $510(2.8 \%)$ & $40(4.0 \%)$ \\
\hline Heart retransplant & $27,013(99.7 \%)$ & $679(2.5 \%)$ & $344(1.9 \%)$ & $46(4.6 \%)$ \\
\hline Congenital heart disease & $27,013(99.7 \%)$ & $501(1.9 \%)$ & $332(1.8 \%)$ & $31(3.0 \%)$ \\
\hline Year of transplant & $27,102(100 \%)$ & 1996 IQR, 1993-2000 & 1996 IQR, 1993-2000 & 1997 IQR, 1993-2000 \\
\hline BMI $\left(\mathrm{kg} / \mathrm{m}^{2}\right)$ & $26,750(98.7 \%)$ & $25.7 \pm 5.0$ & $25.7 \pm 5.1$ & $25.1 \pm 4.7$ \\
\hline $\mathrm{BMI} \geq 35 \mathrm{~kg} / \mathrm{m}^{2}$ & $26,750(98.7 \%)$ & $803(3.0 \%)$ & $520(2.9 \%)$ & $23(2.3 \%)$ \\
\hline Status 1A & $26,360(97.3 \%)$ & $13,400(50.8 \%)$ & $8825(49.8 \%)$ & $603(61.6 \%)$ \\
\hline Status $1 \mathrm{~A}$ or $1 \mathrm{~B}$ & $26,360(97.3 \%)$ & $16,972(64.4 \%)$ & $11,215(63.3 \%)$ & $743(75.9 \%)$ \\
\hline NYHA class IV & $11,666(44.3 \%)$ & $610(5.2 \%)$ & $416(5.2 \%)$ & $21(5.8 \%)$ \\
\hline NYHA class III-IV & $11,666(44.3 \%)$ & $7541(65.6 \%)$ & $5266(65.2 \%)$ & $232(64.3 \%)$ \\
\hline \multicolumn{5}{|l|}{ Life support } \\
\hline Ventilator, n (\%) & $27,102(100 \%)$ & $802(3.0 \%)$ & $536(3.0 \%)$ & $44(4.3 \%)$ \\
\hline Inotropes, $\mathrm{n}(\%)$ & $27,102(100 \%)$ & $11,142(41.1 \%)$ & $7813(43.0 \%)$ & $405(39.9 \%)$ \\
\hline Intra-aortic balloon pump, $\mathrm{n}(\%)$ & $27,102(100 \%)$ & $1510(5.6 \%)$ & $1025(5.6 \%)$ & $61(6.0 \%)$ \\
\hline ECMO, n $(\%)$ & $27,102(100 \%)$ & $44(0.2 \%)$ & $33(0.2 \%)$ & $2(0.2 \%)$ \\
\hline VAD, n $(\%)$ & $27,102(100 \%)$ & $140(0.5 \%)$ & $69(0.4 \%)$ & $7(0.7 \%)$ \\
\hline \multicolumn{5}{|l|}{ Renal function, diabetes, bilirubin } \\
\hline Creatinine clearance & $18,769(69.3 \%)$ & $77.7 \pm 37.9$ & $77.5 \pm 37.5$ & $76.3 \pm 34.4$ \\
\hline Creatinine clearance $<50 \mathrm{~mL} / \mathrm{min}$ & $18,769(69.3 \%)$ & $3486(18.6 \%)$ & $2365(18.4 \%)$ & $145(20.9 \%)$ \\
\hline Dialysis, n (\%) & $18,903(69.7 \%)$ & $586(3.1 \%)$ & $315(2.5 \%)$ & $37(5.2 \%)$ \\
\hline Diabetes, n (\%) & $17,294(63.8 \%)$ & $3170(18.3 \%)$ & $2172(18.5 \%)$ & $108(16.2 \%)$ \\
\hline Serum bilirubin $(\mathrm{mg} / \mathrm{dL})$ & $17,237(63.6 \%)$ & $1.37 \pm 3.49$ & $1.32 \pm 3.07$ & $1.29 \pm 3.39$ \\
\hline Serum bilirubin $\geq 2 \mathrm{mg} / \mathrm{dL}$ ) & $17,237(63.6 \%)$ & $1974(11.5 \%)$ & $1325(11.2 \%)$ & $82(12.6 \%)$ \\
\hline \multicolumn{5}{|l|}{ Hemodynamic parameters } \\
\hline Cardiac index $\left(\mathrm{L} / \mathrm{min} / \mathrm{m}^{2}\right)$ & $17,758(65.5 \%)$ & $2.27 \pm 0.75$ & $2.27 \pm 0.74$ & $2.24 \pm 0.77$ \\
\hline Systolic pulmonary artery pressure $(\mathrm{mm} \mathrm{Hg})$ & $18,272(67.4 \%)$ & $44.4 \pm 15.2$ & $44.4 \pm 15.3$ & $45.6 \pm 14.8$ \\
\hline Mean pulmonary artery pressure $(\mathrm{mm} \mathrm{Hg})$ & $17,853(65.9 \%)$ & $30.2 \pm 10.8$ & $30.1 \pm 10.9$ & $31.3 \pm 10.8$ \\
\hline Pulmonary capillary wedge pressure $(\mathrm{mm} \mathrm{Hg})$ & $18,011(66.5 \%)$ & $20.5 \pm 9.1$ & $20.5 \pm 9.1$ & $21.4 \pm 9.3$ \\
\hline \multicolumn{5}{|l|}{ Donor/operative characteristics } \\
\hline Bicaval & $27,003(99.6 \%)$ & $3601(13.3 \%)$ & $2419(13.4 \%)$ & $135(13.4 \%)$ \\
\hline Organ ischemic time $(\mathrm{h})$ & $25,297(93.3 \%)$ & $2.86 \pm 1.01$ & $2.86 \pm 1.01$ & $2.94 \pm 1.03$ \\
\hline Distance organ transported (miles) & $26,117(96.4 \%)$ & $127 \pm 189$ & $131 \pm 191$ & $119 \pm 188$ \\
\hline Donor age (y) & $27,100(99.9 \%)$ & $30.4 \pm 12.5$ & $30.4 \pm 12.5$ & $30.6 \pm 12.7$ \\
\hline Gender match & $27,102(100 \%)$ & $18,801(69.4 \%)$ & $12,563(69.2 \%)$ & $640(63.0 \%)$ \\
\hline Race match & $27,001(99.6 \%)$ & $18,261(67.6 \%)$ & $12,359(68.3 \%)$ & $639(62.3 \%)$ \\
\hline \multicolumn{5}{|l|}{ PRA activity } \\
\hline Substantial $(\geq 20 \%)$ absolute reduction in PRA activity & $27,102(100 \%)$ & $1244(4.6 \%)$ & $0(0.0 \%)$ & $0(0.0 \%)$ \\
\hline Peak PRA activity $(\%)$ & $27,102(100 \%)$ & $6.6 \pm 17.8$ & $0 \pm 0.0$ & $52.9 \pm 26.8$ \\
\hline Most recent PRA activity $(\%)$ & $27,102(100 \%)$ & $3.7 \pm 13.0$ & $0 \pm 0.0$ & $52.7 \pm 26.8$ \\
\hline
\end{tabular}

$P$ values $<.05$ are in bold. PRA, Panel reactive antibody; BMI, body mass index; NYHA, New York Heart Association; ECMO, extracorporeal membrane oxygenation; VAD, ventricular assist device; $I Q R$, interquartile range. ${ }^{*} P$ value based on analysis of variance. $\dagger P$ value based on chi-square or Student $t$ test analysis. 
TABLE 1. Continued

\begin{tabular}{|c|c|c|c|c|}
\hline \multicolumn{2}{|c|}{$\begin{array}{c}\text { Unadjusted analysis, stratified by peak and } \\
\text { most recent PRA activity }\end{array}$} & \multicolumn{3}{|c|}{ Propensity-matched analysis } \\
\hline $\begin{array}{c}\text { Peak PRA } \geq \mathbf{2 0} \%, \\
\text { substantial reduction in } \\
\text { PRA }(\geq \mathbf{2 0} \%)(\mathbf{n}=\mathbf{1 2 4 4})\end{array}$ & $P^{*}$ & $\begin{array}{c}\text { Peak PRA } \geq 20 \%, \\
\text { trivial reduction in } \\
\text { PRA }(<5 \%)(n=945)\end{array}$ & $\begin{array}{c}\text { Peak PRA } \geq \mathbf{2 0} \%, \\
\text { substantial reduction in } \\
\text { PRA }(\geq \mathbf{2 0} \%)(\mathbf{n}=\mathbf{9 4 5})\end{array}$ & $\boldsymbol{P} \dagger$ \\
\hline $50.6 \pm 11.5$ & $<.001$ & $50.8 \pm 11.4$ & $50.5 \pm 11.8$ & .54 \\
\hline $237(19.1 \%)$ & .02 & $194(20.5 \%)$ & $190(20.1 \%)$ & .82 \\
\hline $841(67.6 \%)$ & $<.001$ & $602(63.7 \%)$ & $599(63.4 \%)$ & .89 \\
\hline $1005(80.8 \%)$ & .01 & $746(78.9 \%)$ & $755(79.9 \%)$ & .61 \\
\hline $167(13.4 \%)$ & $<.001$ & $133(14.1 \%)$ & $124(13.1 \%)$ & .55 \\
\hline $56(4.5 \%)$ & .65 & $50(5.3 \%)$ & $51(5.4 \%)$ & .92 \\
\hline $264(44.6 \%)$ & .10 & $197(43.3 \%)$ & $192(43.2 \%)$ & .99 \\
\hline $585(63.9 \%)$ & .52 & $408(61.9 \%)$ & $421(61.6 \%)$ & .92 \\
\hline $588(47.4 \%)$ & .002 & $443(47.1 \%)$ & $445(47.3 \%)$ & .93 \\
\hline $387(31.2 \%)$ & .03 & $319(33.9 \%)$ & $316(33.6 \%)$ & .88 \\
\hline $58(4.7 \%)$ & $<.001$ & $40(4.3 \%)$ & $42(4.5 \%)$ & .82 \\
\hline $88(7.1 \%)$ & $<.001$ & $45(4.8 \%)$ & $45(4.8 \%)$ & 1.00 \\
\hline $19(1.5 \%)$ & .01 & $17(1.8 \%)$ & $19(2.0 \%)$ & .74 \\
\hline 1997 IQR, 1994-2000 & & 1997 IQR, 1993-2000 & 1997 IQR, 1994-2000 & \\
\hline $26.1 \pm 5.5$ & $<.001$ & $25.3 \pm 4.6$ & $25.4 \pm 4.6$ & .60 \\
\hline $49(4.0 \%)$ & .04 & $22(2.4 \%)$ & $22(2.4 \%)$ & .98 \\
\hline $686(56.7 \%)$ & $<.001$ & $555(60.7 \%)$ & $550(60.2 \%)$ & .81 \\
\hline $916(75.7 \%)$ & $<.001$ & $692(75.7 \%)$ & $690(75.5 \%)$ & .91 \\
\hline $34(6.8 \%)$ & .24 & $20(5.7 \%)$ & $18(4.8 \%)$ & .59 \\
\hline $316(63.5 \%)$ & .70 & $227(65.0 \%)$ & $245(65.9 \%)$ & .82 \\
\hline $47(3.8 \%)$ & .02 & $40(4.2 \%)$ & $38(4.0 \%)$ & .82 \\
\hline $474(38.1 \%)$ & .001 & $371(39.3 \%)$ & $370(39.2 \%)$ & .96 \\
\hline $58(4.7 \%)$ & .29 & $51(5.4 \%)$ & $51(5.4 \%)$ & 1.00 \\
\hline $2(0.2 \%)$ & .98 & $1(0.1 \%)$ & $2(0.2 \%)$ & .56 \\
\hline $17(1.4 \%)$ & $<.001$ & $7(0.7 \%)$ & $10(1.1 \%)$ & .47 \\
\hline $77.5 \pm 37.8$ & .72 & $76.0 \pm 34.5$ & $76.2 \pm 37.2$ & .92 \\
\hline $202(22.4 \%)$ & .004 & $140(21.2 \%)$ & $152(22.6 \%)$ & .54 \\
\hline $53(5.7 \%)$ & $<.001$ & $35(5.2 \%)$ & $34(4.8 \%)$ & .76 \\
\hline $152(17.4 \%)$ & .27 & $104(16.5 \%)$ & $124(18.8 \%)$ & .29 \\
\hline $1.29 \pm 2.81$ & .95 & $1.30 \pm 3.47$ & $1.38 \pm 3.21$ & .68 \\
\hline $90(10.8 \%)$ & .53 & $76(12.3 \%)$ & $71(11.6 \%)$ & .70 \\
\hline $2.30 \pm 0.77$ & .30 & $2.26 \pm 0.77$ & $2.22 \pm 0.72$ & .37 \\
\hline $44.3 \pm 14.9$ & .13 & $45.5 \pm 17.8$ & $45.6 \pm 15.2$ & .88 \\
\hline $30.0 \pm 10.5$ & .02 & $31.2 \pm 10.7$ & $31.2 \pm 10.8$ & .91 \\
\hline $20.5 \pm 9.1$ & .06 & $21.2 \pm 9.2$ & $21.5 \pm 9.2$ & .59 \\
\hline $188(15.2 \%)$ & .20 & $125(13.3 \%)$ & $142(15.1 \%)$ & .27 \\
\hline $2.96 \pm 1.06$ & $<.001$ & $2.94 \pm 1.04$ & $2.97 \pm 1.07$ & .60 \\
\hline $113 \pm 170$ & .002 & $117 \pm 186$ & $118 \pm 179$ & .91 \\
\hline $30.5 \pm 12.3$ & .78 & $30.9 \pm 12.7$ & $30.6 \pm 12.4$ & .60 \\
\hline $867(69.7 \%)$ & $<.001$ & $618(65.4 \%)$ & $617(65.3 \%)$ & .96 \\
\hline $821(66.3 \%)$ & .001 & $604(63.9 \%)$ & $615(65.4 \%)$ & .49 \\
\hline $1244(100 \%)$ & $<.001$ & $0(0.0 \%)$ & $945(100 \%)$ & $<.001$ \\
\hline $56.1 \pm 26.4$ & $<.001$ & $53.4 \pm 26.9$ & $53.5 \pm 27.1$ & .88 \\
\hline $9.9 \pm 16.9$ & $<.001$ & $53.2 \pm 26.8$ & $11.6 \pm 19.1$ & $<.001$ \\
\hline
\end{tabular}


TABLE 2. Multivariable predictors of graft survival according to a Cox proportional hazards regression analysis

\begin{tabular}{|c|c|c|c|c|}
\hline & $\begin{array}{l}\text { Multivariable hazard } \\
\text { ratio }(95 \% \mathrm{CI})\end{array}$ & $P^{*}$ & $\begin{array}{l}\text { Univariate hazard } \\
\text { ratio }(95 \% \mathrm{CI})\end{array}$ & $\boldsymbol{P} \dagger$ \\
\hline \multicolumn{5}{|l|}{ Baseline characteristics } \\
\hline Age at transplant (y) & & & $1.008(1.006-1.009)$ & $<.001$ \\
\hline Age $>60$ y at transplant & $1.20(1.16-1.25)$ & $<.001$ & $1.24(1.19-1.28)$ & $<.001$ \\
\hline Gender (male) & & & $1.01(0.97-1.05)$ & .58 \\
\hline Race (Caucasian) & & & $0.85(0.82-0.89)$ & $<.001$ \\
\hline Race (African-American) & $1.39(1.33-1.46)$ & $<.001$ & $1.31(1.25-1.37)$ & $<.001$ \\
\hline Race (Hispanic) & & & $0.97(0.90-1.04)$ & .39 \\
\hline College education & $0.96(0.92-0.99)$ & .02 & $0.92(0.89-0.96)$ & $<.001$ \\
\hline Insurance (private) & $0.90(0.87-0.94)$ & $<.001$ & $0.86(0.82-0.89)$ & $<.001$ \\
\hline Ischemic dilated cardiomyopathy & $1.20(1.16-1.25)$ & $<.001$ & $1.19(1.15-1.22)$ & $<.001$ \\
\hline Idiopathic dilated cardiomyopathy & & & $0.85(0.83-0.88)$ & $<.001$ \\
\hline Valvular heart disease & & & $0.87(0.79-0.95)$ & .003 \\
\hline Heart retransplant & $1.51(1.37-1.67)$ & $<.001$ & $1.41(1.29-1.56)$ & $<.001$ \\
\hline Congenital heart disease & $1.16(1.02-1.31)$ & .02 & $0.95(0.84-1.08)$ & .20 \\
\hline Year of transplant & $0.97(0.96-0.98)$ & $<.001$ & $0.982(0.978-0.986)$ & $<.001$ \\
\hline BMI $\left(\mathrm{kg} / \mathrm{m}^{2}\right)$ & & & $1.007(1.004-1.010)$ & $<.001$ \\
\hline $\mathrm{BMI} \geq 35 \mathrm{~kg} / \mathrm{m}^{2}$ & $1.19(1.09-1.31)$ & $<.001$ & $1.15(1.05-1.26)$ & .004 \\
\hline Status $1 \mathrm{~A}$ & & & $1.06(1.03-1.10)$ & $<.001$ \\
\hline Status $1 \mathrm{~A}$ or $1 \mathrm{~B}$ & & & $1.01(0.98-1.05)$ & .39 \\
\hline NYHA class IV & & & $0.99(0.89-1.09)$ & .77 \\
\hline NYHA class III-IV & & & $1.01(0.97-1.05)$ & .50 \\
\hline \multicolumn{5}{|l|}{ Life support } \\
\hline Ventilator & $1.47(1.35-1.61)$ & $<.001$ & $1.62(1.49-1.77)$ & $<.001$ \\
\hline Inotropes & & & $1.00(0.96-1.03)$ & .79 \\
\hline Intra-aortic balloon pump & & & $1.19(1.12-1.28)$ & $<.001$ \\
\hline ECMO & $1.60(1.14-2.25)$ & .007 & $2.18(1.56-3.05)$ & $<.001$ \\
\hline VAD & & & $1.00(0.77-1.30)$ & .98 \\
\hline \multicolumn{5}{|l|}{ Renal function, diabetes, bilirubin } \\
\hline Serum creatinine $(\mathrm{mg} / \mathrm{dL})$ & & & $1.026(1.015-1.036)$ & $<.001$ \\
\hline Creatinine clearance $(\mathrm{mL} / \mathrm{min})$ & & & $0.998(0.997-0.999)$ & $<.001$ \\
\hline Creatinine clearance $<50 \mathrm{~mL} / \mathrm{min}$ & $1.13(1.08-1.19)$ & $<.001$ & $1.24(1.19-1.30)$ & $<.001$ \\
\hline Dialysis & $1.19(1.08-1.32)$ & .001 & $1.31(1.18-1.45)$ & $<.001$ \\
\hline Diabetes & $1.15(1.09-1.21)$ & $<.001$ & $1.18(1.12-1.24)$ & $<.001$ \\
\hline Serum bilirubin (mg/dL) & & & $1.006(1.001-1.010)$ & .02 \\
\hline Serum bilirubin $\geq 2 \mathrm{mg} / \mathrm{dL}$ & $1.09(1.03-1.16)$ & .005 & $1.11(1.04-1.18)$ & .001 \\
\hline \multicolumn{5}{|l|}{ Hemodynamic parameters } \\
\hline Cardiac index $\left(\mathrm{L} / \mathrm{min} / \mathrm{m}^{2}\right)$ & & & $0.98(0.96-1.01)$ & .15 \\
\hline Systolic pulmonary artery pressure $(\mathrm{mm} \mathrm{Hg})$ & & & $1.002(1.001-1.003)$ & $<.001$ \\
\hline Mean pulmonary artery pressure $(\mathrm{mm} \mathrm{Hg})$ & & & $1.002(1.000-1.004)$ & .01 \\
\hline Pulmonary capillary wedge pressure $(\mathrm{mm} \mathrm{Hg})$ & & & $1.001(0.999-1.003)$ & .24 \\
\hline \multicolumn{5}{|l|}{ Donor/operative characteristics } \\
\hline Bicaval & & & $0.89(0.84-0.94)$ & $<.001$ \\
\hline Organ ischemic time & $1.05(1.03-1.06)$ & $<.001$ & $1.04(1.02-1.06)$ & $<.001$ \\
\hline Distance organ transported & & & $1.000(1.000-1.001)$ & .08 \\
\hline Donor age & $1.010(1.009-1.011)$ & $<.001$ & $1.011(1.009-1.012)$ & $<.001$ \\
\hline Gender match & $0.95(0.91-0.98)$ & .001 & $0.92(0.89-0.95)$ & $<.001$ \\
\hline Race match & & & $0.92(0.89-0.95)$ & $<.001$ \\
\hline \multicolumn{5}{|l|}{ PRA activity } \\
\hline Substantial $(\geq 20 \%)$ absolute reduction in PRA activity & $0.88(0.80-0.96)$ & .006 & $1.05(0.97-1.13)$ & .22 \\
\hline Peak PRA activity & $1.0032(1.0022-1.0043)$ & $<.001$ & 1.0024 (1.0016-1.0033) & $<.001$ \\
\hline Most recent PRA activity & & & $1.0036(1.0025-1.0047)$ & $<.001$ \\
\hline
\end{tabular}

$P$ values $<.05$ are in bold. $C I$, Confidence interval; BMI, body mass index; $N Y H A$, New York Heart Association; $E C M O$, extracorporeal membrane oxygenation; VAD, ventricular assist device; $P R A$, panel reactive antibody. $* P$ value based on multivariable Cox proportional hazards analysis. $\dagger P$ value based on univariate Cox proportional hazards analysis. 


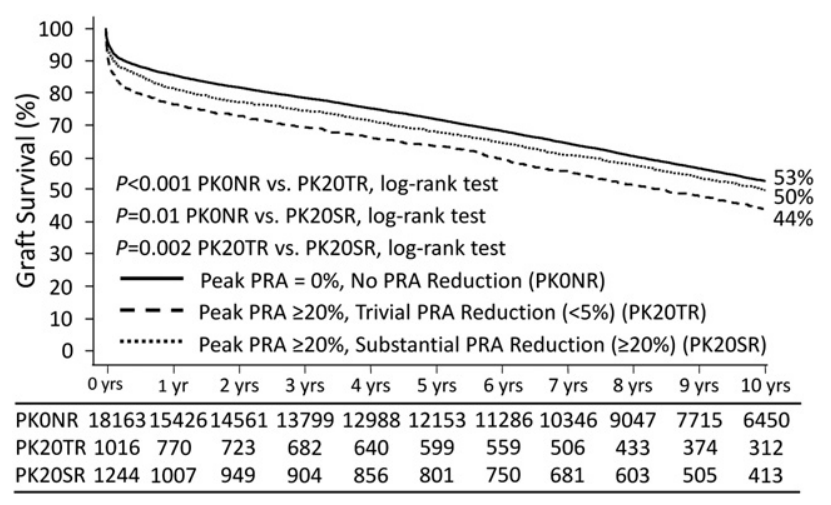

FIGURE 2. Unadjusted Kaplan-Meier estimate of graft survival after heart transplant, stratified by whether the patient had a peak PRA activity of $0 \%$ (ie, no reduction in PRA activity was possible because the peak was $0 \%$ ) (PK0NR), a peak PRA of $20 \%$ or greater but a trivial absolute reduction $(<5 \%)$ in PRA activity (PK20TR), or a peak PRA of $20 \%$ or greater and a substantial absolute reduction ( $\geq 20 \%)$ in PRA activity (PK20SR). $P R A$, Panel reactive antibody.

a peak PRA activity of $20 \%$ or greater who had a significant PRA reduction ( $P \leq .01$ for all comparisons, log-rank).

\section{Propensity-Matched Analysis of Survival After Transplantation}

Among the patients with elevated PRA activity ( $\geq 20 \%)$, matching $1: 1$ by propensity score matched 945 patients who had a trivial reduction in PRA activity with 945 patients who had a significant reduction. Histograms depicting the distribution of propensity scores for patients with an elevated PRA activity $(\geq 20 \%)$ who had a trivial or significant PRA activity reduction are shown for the entire cohort (Figure 3,A) and the propensity-matched subgroup (Figure 3,B). Patient and operation-specific characteristics for the propensity-matched cohort are listed in Table 1. None of the matched preoperative and operative variables were statistically different between groups in the matched cohort, including peak PRA activity. However, the most recent PRA activity (the dependent variable) was 53.2\% $( \pm 26.8 \%)$ in matched patients with a trivial PRA reduction and only $11.6 \%( \pm 19.1 \%)$ in matched patients with a significant PRA reduction $(P<.001)$, indicating that on average, an absolute reduction in PRA activity of more than $40 \%$ was observed in the matched "significant PRA reduction" cohort. A Kaplan-Meier estimate of graft survival (Figure 4) showed a median survival of 120 months in patients with a significant PRA reduction and 103 months in patients with a trivial PRA reduction $(P=.007, \log$-rank $)$.

\section{Early and Late Graft Survival}

The association of a significant PRA reduction with early and late outcomes is shown in Table 3 . The effect of a significant PRA reduction was most notable in early (30-day) outcomes: Patients with a significant PRA reduction had a 50\% decrease in the incidence of death or graft failure (1.15 events per patient year [EPY] vs 1.77 EPY, whereas the event rate was $0.80 \mathrm{EPY}$ in nonsensitized patients with a peak PRA of $0 \%$ ). As expected, the incidence of death or graft failure declined substantially over time, stabilizing at an event rate of 0.07 EPY in patients with trivially reduced PRA and $0.06 \mathrm{EPY}$ in patients with substantially reduced PRA, compared with 0.06 EPY in patients with a peak PRA of $0 \%$.

\section{DISCUSSION}

The current study examined graft survival and potential predictors of graft survival in patients who had a significant reduction in PRA activity and patients who had a trivial reduction in PRA activity. We limited our analysis to an earlier cohort (from 1987 to 2004) of heart transplant recipients to facilitate the assessment of long-term graft survival, to avoid confounding between the 2 eras of PRA recording (MHC class nonspecific vs specific), to minimize the effect of differences in PRA assay (cell-based vs solid-phase), and to avoid confounding the higher PRA activity noted in VAD recipients (of whom there were few in the 1987-2004 era) with graft survival. Cox proportional hazards analysis and propensity-matched techniques were used to minimize selection bias. Our findings suggest that achieving a substantial PRA activity reduction is an independent predictor of graft survival, and that patients who have a substantial PRA reduction attain 10-year graft survival outcomes approaching (but not equal to) those of nonsensitized patients (peak PRA activity of $0 \%$ ).

\section{Panel Reactive Antibody Activity and Significant Reduction in Panel Reactive Antibody Activity as Independent Predictors of Graft Survival}

Our findings suggest that higher peak PRA activity is associated with poorer graft survival in heart transplant recipients (Table 2). Although previous studies have identified elevated PRA activity as a predictor of graft dysfunction in heart transplantation, these studies did not differentiate between peak and most recent PRA activity, nor did they include both peak activity and substantial PRA activity reduction in a multivariable analysis. ${ }^{4,8,9}$ Thus, our analysis produced a novel finding: Peak PRA activity and whether a significant PRA reduction was achieved were independent predictors of graft survival. Of note, in the multivariate analysis, the most recent PRA activity did not predict graft survival independently of peak PRA activity and substantial PRA activity reduction, suggesting that these 2 variables alone adequately account for the effect of elevated PRA activity on graft survival.

\section{Other Predictors of Graft Survival}

In addition to peak PRA activity and a substantial PRA activity reduction, our findings identified many pretransplant patient characteristics and operative variables as predictors 

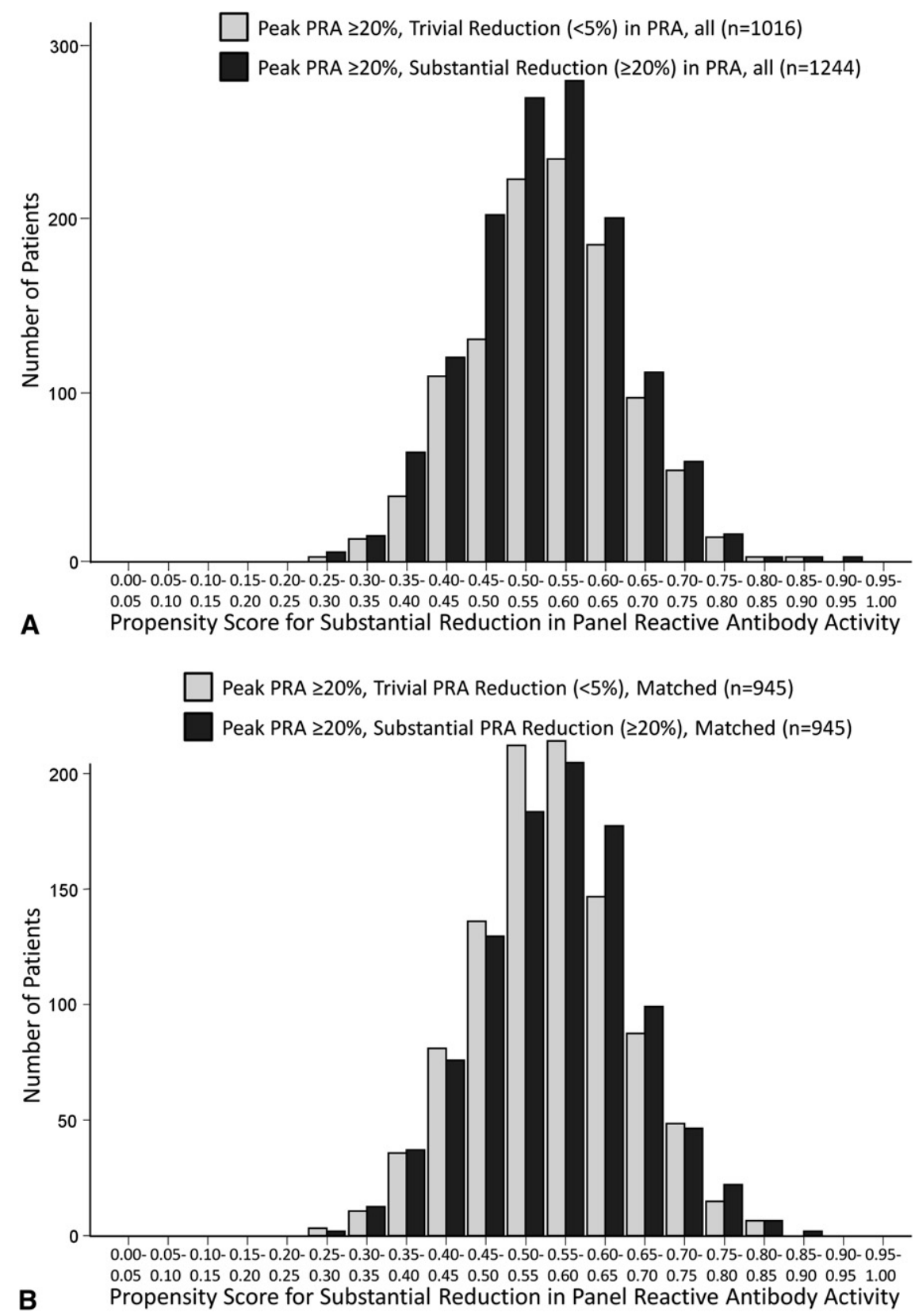

FIGURE 3. Propensity scores for substantial reduction in PRA activity. This propensity score is the probability, computed from baseline variables, that any patient would have a substantial reduction $(>20 \%)$ in PRA activity. A, Propensity scores for all patients who had a trivial reduction (light gray bars) or a substantial reduction (dark gray bars) in PRA activity. B, Propensity score-matched patients (subset of patients from 3A matched by our propensitymatching algorithm) who achieved a trivial (light gray bars) or substantial (dark gray bars) reduction in PRA activity. PRA, Panel reactive antibody.

of graft survival, including age more than 60 years, AfricanAmerican race, college education, private insurance, diagnosis of idiopathic dilated cardiomyopathy, diagnosis of congenital heart disease, undergoing redo heart transplantation, year of transplant, BMI $35 \mathrm{~kg} / \mathrm{m}^{2}$ or greater, ventilator dependence, ECMO support, creatinine clearance less than $50 \mathrm{~mL} / \mathrm{min}$, dialysis requirement, diabetes, serum bilirubin level $2 \mathrm{mg} / \mathrm{dL}$ or greater, organ ischemic time (hours), donor age, and a gender match between organ and recipient
(Table 2). Many of the variables found in this study to independently predict graft survival have been implicated as predictors of post-transplant survival. ${ }^{22,23}$

\section{Unadjusted and Adjusted Post-Transplant Survival}

Our unadjusted Kaplan-Meier survival curves in sensitized and nonsensitized patients suggest that sensitized patients achieving a significant reduction in PRA activity have a survival that approaches (but is not equal to) that 


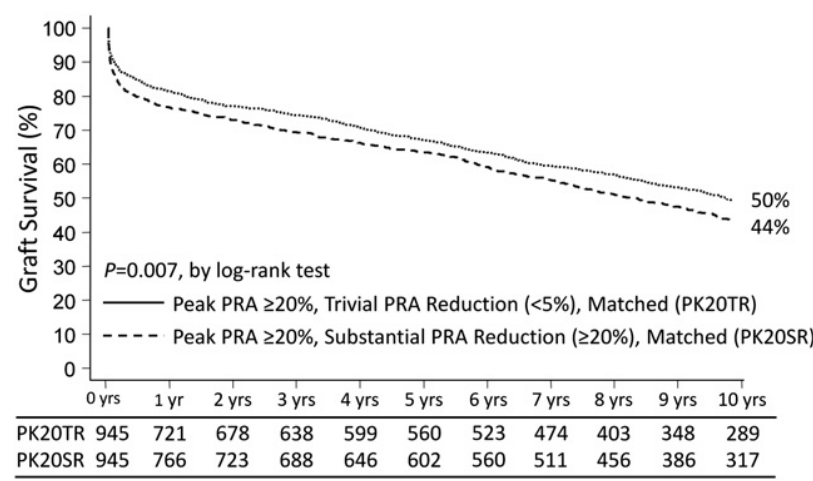

FIGURE 4. Kaplan-Meier estimate of graft survival after heart transplant in a propensity-matched cohort of patients with a peak PRA activity of $20 \%$ or more, stratified by whether a trivial or substantial reduction in PRA activity was achieved. PRA, Panel reactive antibody.

of nonsensitized (peak PRA $=0 \%$ ) patients (Figure 2). However, our unadjusted analysis of graft survival has limited implications given the significant differences among groups in several of the preoperative and operative characteristics (Table 1). We used propensity matching in an effort to account for selection bias, and the effectiveness of our 1:1 matching algorithm is supported by the fact that the matched groups did not significantly differ with regard to any of the matched variables (Table 1). Of note, the Kaplan-Meier survival curves of the propensity-matched groups (sensitized patients who did and did not significantly reduce PRA activity) were similar to our unadjusted comparison; there were only slight differences in median and 10-year survivals (Figure 4). The results of this propensity-matched analysis are confirmed by the main finding of our Cox proportional hazards analysis: A substantial PRA reduction improves graft survival independently of other patient, donor, or operative variables.

\section{Early and Late Graft Survival}

Our analysis of early and late outcomes (Table 3) shows that the primary effect of a preoperative PRA reduction in sensitized patients lies in early (particularly 30-day) outcomes and has a decreasing effect on late outcomes. We suspect that late graft survival is more dependent on the long-term assessment of immunogenicity and the titration of an appropriate immunosuppressive regimen.

\section{Study Limitations}

Our study is limited by its retrospective nature, its susceptibility to hidden bias, and the amount of incomplete data in the UNOS database. We attempted to address these limitations by using multiple imputation to account for missing variables and propensity matching to account for selection bias. Our decision to limit our analysis to a less recent cohort of transplant recipients makes our results less generalizable to today's potential waitlisted patient, for a few reasons. First, the assays used to determine PRA activity in the cohort we studied are dated and have lower sensitivity and specificity for graft survival than more modern techniques. ${ }^{24}$ Second, the PRA activity assessed in this less recent cohort did not differentiate between MHC classes, which simplified our analysis but also made it less applicable to more recent assay results. Third, few patients in our cohort were bridged to transplant with a VAD, whereas this practice is common in today's waitlisted patients; this difference has significant implications regarding PRA activity. ${ }^{25}$ Moreover, no data were available regarding strategies used to reduce PRA activity; therefore, although we strongly recommend PRA reduction in sensitized patients, we are unable to recommend a particular PRAreduction strategy.

\section{CONCLUSIONS}

Our finding that a significant PRA reduction was independently associated with an improvement in graft survival both by Cox proportional hazards modeling (HR, 0.88; CI, $0.80-0.96 ; P=.006)$ and in our propensity-matched Kaplan-Meier survival analysis (17-month improvement in graft survival, $P=.007, \log$-rank) validates current attempts to reduce PRA activity in allosensitized waitlisted patients, and it sets the stage for future analysis of the

TABLE 3. Incidence of death or graft failure at various time intervals after heart transplant

\begin{tabular}{|c|c|c|c|c|c|c|c|c|c|c|c|c|}
\hline \multirow{3}{*}{$\begin{array}{l}\text { Time since } \\
\text { transplant }\end{array}$} & \multicolumn{7}{|c|}{ Unadjusted analysis, stratified by peak and most recent PRA activity } & \multicolumn{5}{|c|}{ Propensity-matched analysis } \\
\hline & \multicolumn{2}{|c|}{$\begin{array}{c}\text { Peak PRA }=0 \%, \\
\text { no reduction in } \\
\text { PRA }(n=18,163) \\
\end{array}$} & \multicolumn{2}{|c|}{$\begin{array}{c}\text { Peak PRA } \geq 20 \%, \\
\text { trivial reduction in } \\
\text { PRA }(<5 \%)(n=1016) \\
\end{array}$} & \multicolumn{2}{|c|}{$\begin{array}{c}\text { Peak PRA } \geq 20 \%, \\
\text { substantial reduction in } \\
\text { PRA }(\geq \mathbf{2 0} \%)(n=1244)\end{array}$} & \multirow[b]{2}{*}{$P^{*}$} & \multicolumn{2}{|c|}{$\begin{array}{c}\text { Peak } \mathrm{PRA} \geq \mathbf{2 0} \%, \\
\text { trivial reduction in } \\
\text { PRA }(<\mathbf{5} \%)(\mathrm{n}=\mathbf{9 4 5})\end{array}$} & \multicolumn{2}{|c|}{$\begin{array}{c}\text { Peak PRA } \geq 20 \%, \\
\text { substantial reduction in } \\
\text { PRA }(\geq \mathbf{2 0} \%)(n=945) \\
\end{array}$} & \multirow[b]{2}{*}{$\boldsymbol{P} \dagger$} \\
\hline & No. $(\%)$ & EPY & No. $(\%)$ & EPY & No. $(\%)$ & EPY & & No. $(\%)$ & EPY & No. $(\%)$ & EPY & \\
\hline $0-30 \mathrm{~d}$ & $1136(6.3)$ & 0.80 & $133(13.1)$ & 1.77 & $109(8.8)$ & 1.15 & $<.001$ & $122(12.9)$ & 1.73 & $88(9.3)$ & 1.22 & .01 \\
\hline $31-90 \mathrm{~d}$ & $543(3.2)$ & 0.20 & $47(5.4)$ & 0.34 & $45(4.0)$ & 0.25 & .001 & $46(5.6)$ & 0.35 & $36(4.2)$ & 0.26 & .19 \\
\hline $91-180 \mathrm{~d}$ & $354(2.2)$ & 0.09 & $25(3.0)$ & 0.12 & $27(2.5)$ & 0.10 & .22 & $24(3.1)$ & 0.13 & $21(2.6)$ & 0.11 & .52 \\
\hline $181-365 \mathrm{~d}$ & $481(3.0)$ & 0.06 & $32(3.9)$ & 0.08 & 49 (4.6) & 0.09 & .005 & $28(3.7)$ & 0.08 & $31(3.9)$ & 0.08 & .86 \\
\hline$>1 \mathrm{y}$ & $7563(49.0)$ & 0.06 & $414(53.7)$ & 0.07 & $481(47.9)$ & 0.06 & .06 & $392(53.7)$ & 0.07 & $372(48.7)$ & 0.06 & .14 \\
\hline
\end{tabular}

$P R A$, Panel reactive antibody; $E P Y$, events per patient year after heart transplant. $* P$ value is based on comparison among 3 groups by the log-rank test. $\dagger P$ value is based on comparison between 2 groups by the log-rank test. 
effects of PRA reduction in a more recent cohort of transplant recipients.

Stephen N. Palmer, PhD, ELS, contributed to the editing of the manuscript.

\section{References}

1. Velez M, Johnson MR. Management of allosensitized cardiac transplant candidates. Transplant Rev (Orlando). 2009;23:235-47.

2. Kerman RH. Understanding the sensitized patient. Heart Fail Clin. 2007;3:1-9.

3. Patel R, Terasaki PI. Significance of the positive crossmatch test in kidney transplantation. N Engl J Med. 1969;280:735-9.

4. Smith JD, Danskine AJ, Laylor RM, Rose ML, Yacoub MH. The effect of panel reactive antibodies and the donor specific crossmatch on graft survival after heart and heart-lung transplantation. Transpl Immunol. 1993;1:60-5.

5. Betkowski AS, Graff R, Chen JJ, Hauptman PJ. Panel-reactive antibody screening practices prior to heart transplantation. J Heart Lung Transplant. 2002;21: 644-50.

6. Tyan DB. New approaches for detecting complement-fixing antibodies. Curr Opin Organ Transplant. 2012;17:409-15.

7. Terasaki PI, Kreisler M, Mickey RM. Presensitization and kidney transplant failures. Postgrad Med J. 1971;47:89-100.

8. Lavee J, Kormos RL, Duquesnoy RJ, Zerbe TR, Armitage JM, Vanek M, et al Influence of panel-reactive antibody and lymphocytotoxic crossmatch on survival after heart transplantation. J Heart Lung Transplant. 1991;10:921-30.

9. Nwakanma LU, Williams JA, Weiss ES, Russell SD, Baumgartner WA, Conte JV. Influence of pretransplant panel-reactive antibody on outcomes in 8,160 heart transplant recipients in recent era. Ann Thorac Surg. 2007;84: 1556-63.

10. Ting A, Hasegawa T, Ferrone S, Reisfeld RA. Presensitization detected by sensitive crossmatch tests. Transplant Proc. 1973;5:813-7.

11. Zachary AA, Klingman L, Thorne N, Smerglia AR, Teresi GA. Variations of the lymphocytotoxicity test: an evaluation of sensitivity and specificity. Transplantation. 1995;60:498-503.

12. Bradley JA, Baldwin WM, Bingaman A, Ellenrieder C, Gebel HM, Glotz D, et al. Antibody-mediated rejection-an ounce of prevention is worth a pound of cure. Am J Transplant. 2011;11:1131-9.

13. Stehlik J, Islam N, Hurst D, Kfoury AG, Movsesian MA, Fuller A, et al. Utility of virtual crossmatch in sensitized patients awaiting heart transplantation. $J$ Heart Lung Transplant. 2009;28:1129-34.

14. Cecka JM. Calculated PRA (CPRA): the new measure of sensitization for transplant candidates. Am J Transplant. 2010;10:26-9.

15. Mahle WT, Tresler MA, Edens RE, Rusconi P, George JF, Naftel DC, et al. Allosensitization and outcomes in pediatric heart transplantation. J Heart Lung Transplant. 2011;30:1221-7.

16. Leech SH, Lopez-Cepero M, LeFor WM, DiChiara L, Weston M, Furukawa S, et al. Management of the sensitized cardiac recipient: the use of plasmapheresis and intravenous immunoglobulin. Clin Transplant. 2006;20:476-84.

17. Kobashigawa JA, Patel JK, Kittleson MM, Kawano MA, Kiyosaki KK, Davis SN, et al. The long-term outcome of treated sensitized patients who undergo heart transplantation. Clin Transplant. 2011;25:E61-7.

18. Pisani BA, Mullen GM, Malinowska K, Lawless CE, Mendez J, Silver MA, et al. Plasmapheresis with intravenous immunoglobulin $\mathrm{G}$ is effective in patients with elevated panel reactive antibody prior to cardiac transplantation. J Heart Lung Transplant. 1999;18:701-6.

19. Morrow WR, Frazier EA, Mahle WT, Harville TO, Pye SE, Knecht KR, et al. Rapid reduction in donor-specific anti-human leukocyte antigen antibodies and reversal of antibody-mediated rejection with bortezomib in pediatric heart transplant patients. Transplantation. 2012;93:319-24.

20. Massad MG, Cook DJ, Schmitt SK, Smedira NG, McCarthy JF, Vargo RL, et al. Factors influencing HLA sensitization in implantable LVAD recipients. Ann Thorac Surg. 1997;64:1120-5.

21. Drakos SG, Kfoury AG, Kotter JR, Reid BB, Clayson SE, Selzman CH, et al. Prior human leukocyte antigen-allosensitization and left ventricular assist device type affect degree of post-implantation human leukocyte antigen-allosensitization. J Heart Lung Transplant. 2009;28:838-42.

22. Weiss ES, Allen JG, Arnaoutakis GJ, George TJ, Russell SD, Shah AS, et al. Creation of a quantitative recipient risk index for mortality prediction after cardiac transplantation (IMPACT). Ann Thorac Surg. 2011;92:914-22.
23. Weiss ES, Allen JG, Patel ND, Russell SD, Baumgartner WA, Shah AS, et al. The impact of donor-recipient sex matching on survival after orthotopic heart transplantation: analysis of 18000 transplants in the modern era. Circ Heart Fail. 2009;2:401-8

24. Saidman SL. Histocompatibility testing for highly sensitized transplant candidates. Transplant Proc. 2007;39:673-5.

25. Arnaoutakis GJ, George TJ, Kilic A, Weiss ES, Russell SD, Conte JV, et al. Effect of sensitization in US heart transplant recipients bridged with a ventricular assist device: update in a modern cohort. J Thorac Cardiovasc Surg. 2011;142: 1236-45. 1245.e1.

\section{Discussion}

Dr Joseph C. Cleveland (Aurora, Colo). The management of an allosensitized potential cardiac transplant recipient remains highly anecdotal and center specific as outlined in the presentation. In essence, each center possesses its own witch's brew-the treatment to reduce PRA levels, including plasmapheresis, intravenous immunoglobulin, and some novel newer agents.

This analysis by Schaffer and colleagues is important and seeks to provide guidance for managing these allosensitized patients. The findings of the present study are novel and important. Both the peak PRA level and the inability to reduce the PRA level by less than $20 \%$ leads to an inferior allograft survival, at least in this UNOS registry over a 10-year period. I applaud the authors' use of propensity matching to try to equal as much as you can, make the patient comorbidities as equitable as possible. I have 3 questions.

My first question pertains to the obvious choice of time periods for analysis as you outlined. The way that we now analyze PRA in the cell-based Luminex (Austin, Tex) technology is so different than in the prior era of 1987-2004. I acknowledge your desire to keep a homogeneous patient population, but why not focus on the period of transplantation that we are now dealing with, from 2004 to 2010. What made you want to look from 1987 to 2004? What about a patient who has a PRA of a mean fluorescent intensity of 2000 units in one of these cell-based technologies: Do you think your data really will help us deal with that type of patient?

Dr Schaffer (Stanford, Calif). To be honest, the real reason we started this study of the "older" era was to simplify our analysis and to serve as a starting point for future study. If we could prove that a reduction in MHC class I PRA levels before transplant independently improved long-term survival in the "older era" cohort, we would have a good starting point to try and differentiate between the different assays used in the more recent PRA era. This study serves as our starting point. We now know that a PRA reduction independently improves post-transplantation survival. Now we have to try and figure out how it works in the more recent era. Factors that complicate this analysis are that more recent technologies assess both class 1 and 2 PRA percentages. Now we can try and assess whether a reduction in a particular MHC class matters more, and to try to determine how the different methods of measuring PRA level in the more recent era affects the documented levels of PRA activity.

Dr Cleveland. Fair enough. So you report your primary end graft is allograft survival. One of the potential risks to aggressive desensitization protocols is the occurrence of excess mortality after transplantation that is due to infectious causes. Was overall patient survival between these groups analyzed? Do you have those data? 
Dr Schaffer. I do not have the curve to show you, but from our experience the post-transplant graft survival and mortality curves are similar. The study's primary end point is a composite of all cause mortality and graft failure, which we believe is the most appropriate end point for this analysis.

Dr Cleveland. You do not think you saw an excess signal indicating mortality from infectious causes at least as best you could?

Dr Schaffer. Our study did not specifically assess causes of death, only time to graft failure or death.

Dr Cleveland. Last, as you acknowledge, there were not a lot of VAD recipients in this cohort, but one of the principal methods whereby patients develop elevated PRA levels are those that require a left VAD as a bridge to transplantation. The Johns Hopkins group published an analysis from the same UNOS thoracic registry. They stratified left VAD recipients with PRA of $0 \%$ versus $25 \%$ and observed no difference in rejection rates for the first year after cardiac transplantation and no difference in overall patient survival. Can you put your study in perspective with their data? How do your data and analysis perhaps differ from their study and other ways that patients might be treated differently to desensitize them if they are a VAD recipient?

Dr Schaffer. I suspect the Hopkins group, when they were creating their study, probably ran into the same troubles that we did in trying to decide how to differentiate between a peak and a most recent PRA level when you have 2 different values for the 2 different MHC classes. I suspect that is why they limited their analysis to just the most recent PRA levels. As to differentiating ours, I think our analysis gives you a good idea what to expect for the non-VAD recipient, and theirs obviously is limited to VAD recipients. Their study does make the important point as to how useful PRA levels are in the population with VADs. We hope to assess this in our future work assessing the more recent era.

Dr Cleveland. One last minor follow-up question in terms of the Kaplan-Meier curves. It looks like the initial first year is really where this difference is. Are those slopes truly similar or is there a late risk that these patients might incur by having elevated PRA?
Dr Schaffer. In our analysis there is a statistically significant difference, but it is tiny. I forget the event rate per se, but it is so small as to not be clinically relevant. All of that risk really comes in the first year and most in the first 6 months.

Dr Cleveland. Nice presentation.

Dr Timothy Icenogle (Spokane, Wash). I rise to translate for the audience what I think is an enormous contribution to medical science. What these therapies are aimed at are basically deleting immunologic memory, that is, to delete the memory cells of a previous exposure to somebody else's tissues. The new studies that you described are highly sensitive and specific. What you have shown is that by deleting immunologic memory or at least a portion of immunologic memory, there has been a reduction in bad outcomes in transplantation. This translates well beyond the world of transplantation to the world of autoimmune diseases, including type 1 diabetes, amyotrophic lateral sclerosis, multiple sclerosis, and traumatic chronic encephalopathy. If we are able to delete immunologic memory, and in the world of transplantation led by young surgeons such as yourself, we can now explore ways to delete immunologic memory, this will have huge implications for the rest of medicine. In your evaluation of all of these therapies, there has been considerable improvement. In your studies, were you able to find any therapies that were perhaps more successful than other therapies in deleting immunologic memory?

Dr Schaffer. You have identified the biggest problem with our study. I must not have made it clear during my talk, but the huge limitation of the UNOS database is that we do not have the underlying treatment as to what these patients received. We just know that they had a high PRA level and that it was substantially reduced at the time of transplant. I wish I had those data (treatment type). To my knowledge, those data are not prospectively collected in the UNOS database. That is obviously the key to this, and we do not have that right now, but in newer data sets in the more recent era we do have some of those data. We will be trying to assess that in future studies. 